Esta obra forma parte del acervo de la Biblioteca Jurídica Virtual del Instituto de Investigaciones Jurídicas de la UNAM

\title{
LOS MÉTODOS DE ELECCIÓN DE CANDIDATOS EN MÉXICO: UNA PROPUESTA DE INGENIERÍA POLÍTICA DEMOCRATIZADORA
}

\author{
Francisco José de ANDREA SÁNCHEZ ${ }^{1}$
}

\section{Sumario:}

I. Conceptos esenciales en materia de métodos de selección de candidatos y líderes políticos

II. Elecciones internas democráticas y sus efectos sobre los partidos políticos estadounidenses

III. Algunas consideraciones finales

RESUMEN: El presente ensayo tiene un objetivo múltiple: servir como punto de partida para eventuales propuestas de reforma de la vida partidaria interna de los partidos políticos mexicanos que implementen la celebración institucional, periódica y permanente de elecciones primarias internas como elecciones preliminares que antecedan a las elecciones generales para seleccionar a candidatos a cargos de elección popular de nivel municipal, estatal y federal; también, dar un giro novedoso a los análisis en materia de las elecciones internas de los partidos políticos, para enfatizar las principales fortalezas de las elecciones primarias; además, arrojar luces sobre la segunda vertiente de la democracia interna de los partidos políticos contemporáneos que es la selección de sus cuadros directivos; asimismo, demostrar que las elecciones primarias disminuyen la posibilidad de narcocandidatos que accedan exitosamente al poder a través de elecciones constitucionales, "lavando" así su capital político. Por último, este trabajo enfatiza que los partidos políticos son instituciones fundamentales para la estabilidad política y social de un país, y del propio sistema político de partidos.

Palabras clave: Democracia interna de los partidos, candidatos políticos, campañas electorales, narcopolítica, partidos políticos.

ABstRact: Perhaps the main obstacle to achieve a true and definitive democratization of Mexico's political parties and in fact the political system as a whole is the pending exercise

\footnotetext{
${ }^{1}$ Investigador del Instituto de Investigaciones Jurídicas de la UNAM.
} 
Esta obra forma parte del acervo de la Biblioteca Jurídica Virtual del Instituto de Investigaciones Jurídicas de la UNAM www.juridicas.unam.mx

DOI: http://dx.doi.org/10.22201/iij.24487910en2016.9.10096

of political and social engineering needed to reform the current elitist and undemocratic methods in place for decades and replace the convention, caucus and "dedazo" methods with a system of internal primary elections which allow a wider participation of grass roots organizations and the populace in general thus legitimizing the results and outcomes of both internal party and congressional and presidential elections. In this essay, we analyze the evolution and current status of primary elections in the USA as a solid background which will shed light on the aforesaid weaknesses of the Mexican political and electoral system.

Keywords: Political parties, narcopolitics, political candidates, campaigns, primary elections.

\section{CONCEPTOS ESENCIALES EN MATERIA DE ELECCIONES PRIMARIAS}

\section{Contextualización del tema y concepto de elecciones primarias}

Este ensayo tiene como objetivo apuntalar nuestra principal propuesta académica de que en el futuro los partidos políticos mexicanos - al seleccionar a sus candidatos políticos para los diversos puestos de elección popular - se inclinen por el método de las elecciones primarias - bajo el modelo clásico estadounidense- por encima de las convenciones, encuestas o "dedazos", que en el entorno político y social nacional actuales — casos Tlatlaya y Ayotzinapa - generan conflictos innecesarios y reducen la legitimidad de origen de los candidatos políticos emanados de dichos procesos, todo lo cual repercute también en la eficiencia y calidad de los procesos electorales generales posteriores.

La elección primaria es un método o procedimiento a través del cual los partidos políticos contemporáneos seleccionan a sus candidatos a puestos de elección popular. Los ganadores de las elecciones primarias compiten posteriormente contra los candidatos de otros partidos en la elección general. En el anterior sentido, las elecciones primarias de hechos son elecciones preliminares a la elección general. Cabe resaltar que, fuera de Estados Unidos de América, es común entre los no especialistas — tratándose de elecciones primarias - pensar fundamentalmente en las elecciones primarias presidenciales, pero cabe aclarar que, aunque menos analizadas y estudiadas, las elecciones primarias legislativas, y para cargos como alcaldías y presidencias municipales, son ya desde hace tiempo de uso generalizado en los Estados Unidos, y en general han dado extraordinarios resultados en la democratización de los procesos de selección, tanto de los candidatos a puestos de elección popular como de líderes de los propios 
Esta obra forma parte del acervo de la Biblioteca Jurídica Virtual del Instituto de Investigaciones Jurídicas de la UNAM

partidos políticos.

En el anterior sentido, cabe especular que el ejemplo reciente y extremo de un narcocandidato que ganó una elección en una demarcación importante en México es el de José Luis Abarca, quien en 2012 fue postulado por la coalición PRD-Convergencia para el cargo de presidente municipal de Iguala, Guerrero. El proceso de selección de Abarca como candidato consistió fundamentalmente en la generación de una dinámica de apoyo local y nacional apuntalada por la corriente Nueva Izquierda del PRD, específicamente del líder local Sebastián de la Rosa Peláez. ${ }^{2}$ Este caso es significativo y tiene importancia para nuestro estudio, porque de haber existido en México de manera regulada —en los ámbitos local y federal - un método o procedimiento de selección de candidatos basado en elecciones primarias previas o preliminares a la elección general, al interior de cada partido, en el caso de Iguala, habría sido mucho más difícil que un empresario ligado a la delincuencia organizada hubiera pasado los filtros de métodos de selección más democráticos y abiertos al escrutinio público.

Para fortalecer nuestra argumentación, es útil el contraste del proceso político que se gestó en la última elección del alcalde de Nueva York, Bill de Blasio, quien sorpresivamente y a contracorriente, primero ganó la elección primaria interna del Partido Demócrata venciendo a William Thompson Jr., quien era el favorito de la nomenclatura demócrata, y quien ya había sido candidato en 2009 en la elección general, perdiendo ante el alcalde Michael Bloomberg. Posteriormente, De Blasio derrotó en la elección general al republicano Joe Lhota, quien estaba apoyado por figuras políticas como Rudolph Giuliani y los tres principales periódicos de Nueva York, así como por poderosos grupos de la comunidad judía de Nueva York. ${ }^{3}$ La sorpresiva victoria para De Blasio se apuntaló en una coalición plural de intereses populares basados en grupos disímbolos y minoritarios pero muy importantes en número y dinamismo ya integrados en una coalición. En resumen, en la elección del alcalde de la ciudad $\mathrm{Alfa}^{4}$ más poderosa del mundo, en términos económicos, prevaleció un candidato apoyado en su popularidad con el electorado y sus cualidades personales y políticas como hombre liberal, preparado, capaz e incorruptible, por encima de candidatos apoyados por grupos

\footnotetext{
${ }^{2}$ www.excelsior.com.mx/nacional/2014/10/07/985546.

3 "For New York Mayor", The New York Times, 24 de agosto de 2013.

${ }^{4}$ GAWC Research Bulletins, GAWC, Loughborough University, 28 de julio de 1999.
} 
Esta obra forma parte del acervo de la Biblioteca Jurídica Virtual del Instituto de Investigaciones Jurídicas de la UNAM www.juridicas.unam.mx

DOI: http://dx.doi..org/10.22201/iij.24487910e.2016.9.10096

con factores de interés vinculados al gran capital.

\section{Evolución y desarrollo de la elección primaria directa}

La elección primaria es una institución netamente estadounidense, surgida en la era de la reforma política a finales del siglo XIX y principios del XX. La primera elección primaria estatal se llevó a cabo en 1899 en Minnesota y la primera elección primaria presidencial se organizó en 1901 en Florida. ${ }^{5}$

Después de la génesis de los partidos políticos, claramente distinguibles bajo la definición contemporánea de partido, que empezaron a surgir a finales del siglo XIX, un aspirante a una posición de elección popular acostumbraba obtener la nominación de su partido a través de una junta o reunión de líderes de partido (caucus) o de una convención en su pueblo, municipio, ciudad, distrito o estado. Ocasionalmente, dichos procesos se regulaban bajo la legislación de cada entidad federativa, pero, con más frecuencia, dicha dinámica quedaba en manos de los propios partidos políticos.

La primera elección primaria directa se efectuó en el condado de Crawford, Pennsylvania, por parte de los demócratas en $1842 .{ }^{6}$ Las primeras leyes estatales que se refirieron a las elecciones primarias fueron aprobadas en California y en Nueva York en 1866, ${ }^{7}$ aunque cabe destacar que hasta el final del siglo XIX todas las leyes sobre elecciones primarias aprobadas por los estados eran opcionales o cubrían únicamente áreas geográficas limitadas. A finales del siglo XIX, dos eventos condujeron a una elección primaria estatal obligatoria. El primero fue la introducción del sufragio secreto o "australiano", usado por primera vez en Massachusetts en $1888,{ }^{8}$ el sufragio secreto implicaba que un elector podía votar en forma verdaderamente libre y sin inhibiciones al seleccionar a los candidatos de un partido político. El segundo hecho fue la ola de rechazo hacia el sistema de convención bajo el cual -aunque era regulado por el derecho local- los llamados jefes políticos y los intereses creados, que buscaban el provecho personal, usaban todas las tácticas y estrategias imaginables. Cabe señalar que

\footnotetext{
${ }^{5}$ http://aceproject.org/ace-en/topics/pc/pcy/pcy usa\#.

${ }^{6}$ http://sites.allegheny.edu/cpp/voting-guidelines/primary-election/.

${ }^{7}$ http://history.stackexchange.com/questions/1682/why-did-the-political-parties-in-the-us-give-up-powertorun-primary-elections-t.
}

${ }^{8}$ http://homepage.cs.uiowa.edu/ jones/voting/pictures/. 
Esta obra forma parte del acervo de la Biblioteca Jurídica Virtual del Instituto de Investigaciones Jurídicas de la UNAM www.juridicas.unam.mx

resulta interesante resaltar la interrogante de por qué en México la sociedad sigue tolerando los esquemas de las convenciones nacionales, cuando en otras latitudes desde el siglo XIX la sociedad civil estadounidense ya había detectado las numerosas patologías que emanan del sistema de convenciones que per se facilita la manipulación por parte de las nomenclaturas de los partidos políticos.

Ahora bien, volviendo al contexto estadounidense, cabe resaltar que los llamados "progresistas" consideraban a la primaria directa como una cura inmediata para los abusos arriba citados. Así pues, como ya apuntamos anteriormente, en una Ley de 1899, perfeccionada en 1901, el Estado de Minnesota instituyó el primer sistema de elecciones primarias obligatorias a nivel estatal, ubicando a la primaria en el mismo plano que una elección general, y estableciendo su aplicación uniforme en todo el estado.

Las nominaciones de primaria directa para poder aspirar a puestos locales y estatales ha predominado en Estados Unidos de América desde la década de los años veinte del siglo XX. En algunos estados, los partidos políticos apoyan y respaldan a sus candidatos antes de la primaria, ya sea a través de convenciones o de las acciones de los comités estatales. La convención de respaldo o apoyo (endorsing convention) es una forma de establecer una responsabilidad partidaria en la conformación de las fórmulas de candidatos propuestos, a la vez que se le deja la decisión final a los propios electores (la llamada endorsing convention podría ser un método intermedio aceptable para los grandes partidos políticos mexicanos, puesto que la nomenclatura mantiene una dosis de control sobre el proceso compartido con la base partidista y electoral). En algunos estados de la unión americana, quien pierde en el nivel de la convención estatal o local, puede "rentar" nuevamente al ganador en una elección primaria del partido lo que evidentemente democratiza aún más el proceso político.

\section{Las primarias frente a las convenciones}

Así pues, hoy día podemos decir que la postura de ciudadanos comunes y de académicos de que la figura de la primaria directa propiciaría una solución a las patologías del método de nominación "a puerta cerrada" ha sido ampliamente respaldada en los hechos. Cabe destacar que recientemente el debilitamiento y la decadencia de las maquinarias políticas atrincheradas en estructuras de poder tradicionales a nivel estatal y en las grandes ciudades estadounidenses, durante el siglo XX, hubiera sido mucho más lento, sino imposible, de no haber existido la elección primaria que ofrecía una forma de 
Esta obra forma parte del acervo de la Biblioteca Jurídica Virtual del Instituto de Investigaciones Jurídicas de la UNAM www.juridicas.unam.mx

desalojar a grupos, facciones y mafias de corruptos del gobierno. Asimismo, cabe enfatizar también que muchos de los líderes legislativos y estatales estadounidenses más importantes -incluyendo por supuesto al actual presidente Obama-, y que alcanzaron el poder político en el siglo XX y el XXI, hubieran tenido escasas posibilidades y opciones de crecimiento político bajo un sistema de convención.

Por otro lado, no podemos dejar de reconocer que la primaria directa ocasionalmente ha llevado a los abusos y excesos que sus enemigos y críticos predijeron —la nominación de candidatos populares y de relumbrón, quienes en la práctica fueron incompetentes y débiles-, así como a un debilitamiento del liderazgo responsable dentro de los partidos políticos establecidos. Por otra parte, la primaria directa tampoco ha eliminado todas las influencias nocivas asociadas con el sistema de nominación. De esta forma, hay que reconocer que las campañas relacionadas con las elecciones primarias son extremadamente costosas, y los candidatos con frecuencia se ven obligados a buscar financiamiento de los mismos intereses especiales que controlaban a las convenciones partidarias en el pasado.

En Estados Unidos de América, los simpatizantes del sistema de convención rara vez han podido contener la oleada popular que exige leyes que establezcan primarias directas como el método preferido para lograr una democracia interna efectiva. En aquellos lugares en los cuales el sistema de convención se ha conservado, algunos de los abusos de poder más reprobables del siglo XIX han reaparecido. Por ejemplo, hasta que una primaria fue instituida en Nueva York en 1968, los candidatos estatales eran seleccionados a través de una convención, y frecuentemente candidatos que carecían de un apoyo estatal razonable eran los seleccionados. Existen frecuentes acusaciones de que los candidatos eran en realidad los títeres de las nomenclaturas políticas.

\section{Las elecciones primarias abiertas vs. Elecciones}

\section{primarias cerradas}

Ahora bien, una vez que se logra establecer un sistema de elecciones primarias directas, la cuestión clave a menudo es la del "acceso" propiamente dicho a la votación en la elección primaria. ¿Acaso debe existir un sistema "de elección primaria abierta", bajo la cual cualquier elector puede solicitar participar en la primaria de cualquier partido? 0 bien, ¿debe existir una "elección primaria cerrada" bajo la cual la participación está limitada a personas que han registrado afiliación con el partido en una fecha anterior a 
Esta obra forma parte del acervo de la Biblioteca Jurídica Virtual del Instituto de Investigaciones Jurídicas de la UNAM www.juridicas.unam.mx

la propia elección? Quienes apoyan la elección primaria abierta, argumentan que un ciudadano no debe de ser obligado a relevar públicamente su afiliación partidaria, y que se le debería otorgar la oportunidad para votar en la contienda primaria que éste considere más significativa e importante. Por otro lado, también resulta conveniente, argumentan algunos, el excluir a lectores "independientes" del proceso de nominación a través de un sistema de elección primaria cerrada. La elección primaria cerrada, por otro lado, es favorecida por quienes desean tener partidos políticos unificados, disciplinados, bajo el argumento de que con la elección primaria abierta es muy factible que los adherentes de cualquier partido de oposición "asalten" las elecciones primarias de los partidos rivales, con el propósito de nominar a un candidato convenientemente débil.

Ahora bien, debido a que son precisamente los políticos profesionales -que lógicamente apoyan a la institución del partido político- quienes redactan las leyes electorales, ya sea como legisladores o como hombres "fuertes" del sistema político, la gran mayoría de los estados utilizan la primaria cerrada. Sin embargo, cabe aclarar que algunos estados utilizan un "sistema de verificación" en lugar del requisito de la afiliación al partido. De esta forma, a un elector normalmente se le facilita el acceso a la boleta electoral de la elección primaria del partido que éste desee, pero si un observador de casilla decide impugnarlo, el elector puede ser requerido para hacer una declaración jurada escrita en el sentido de que votó por una mayoría de los candidatos del partido en la última elección, o que lo hará en la próxima elección. Debido a que el voto es secreto, el llamado sistema de verificación deja la decisión a la conciencia del elector individual y en la realidad se acerca mucho a la práctica de una primaria abierta.

Actualmente, únicamente 19 estados de la unión americana tienen sistema de "primarias abiertas". ${ }^{9}$ Es interesante consultar la lista completa de dichos estados con elecciones primarias abiertas, cerradas $\mathrm{u}$ otras modalidades. ${ }^{10} \mathrm{El}$ Estado de Washington, en el pasado reciente, decidió ir un paso más adelante con su muy original jung/e primary -elección primaria tipo "jungo"- bajo el cual el elector, sin distingo de afiliación partidaria, tenía libertad para ir y venir entre los principales partidos políticos para seleccionar a un candidato en cada puesto. Cabe destacar que en 2001, la Suprema Corte estadounidense declaró inconstitucional la jungle primary en el caso CA

\footnotetext{
${ }^{9}$ http://www.daylypaul.com/158941/2012-open-primary-estates-the-key-to-ron-pauls-republican-nomination.

${ }^{10}$ http://grassrootsidgob.wordpress.com/list-of-states-with-open-and-closed-primaries/.
} 
Esta obra forma parte del acervo de la Biblioteca Jurídica Virtual del Instituto de Investigaciones Jurídicas de la UNAM www.juridicas.unam.mx

DOI: http://dx.doi.org/10.22201/iij_.24487910e_2016.9.10096

Democratic Party vs. Jones. ${ }^{11}$

Durante muchos años (1913 a 1959), el Estado de California contemplaba un sistema de registro cruzado (cross-filing). ${ }^{12}$ De esta forma, un candidato podía participar en elecciones primarias en ambos partidos, y, por lo tanto, tener asegurada la elección si lograba la victoria en ambas, como lo hizo el gobernador republicano Earl Warren, en 1946.

\section{Las fechas}

Cada estado puede establecer las fechas de celebración de sus primarias como mejor le plazca. Existe un listado completo de las fechas de las primarias en todos los estados de la unión americana para $2014 .{ }^{13}$ La mayoría de las primarias para puestos legislativos o estatales se celebran en mayo, junio o en septiembre de cada año par. ${ }^{14}$

\section{Acceso a la boleta electoral}

Existen tres métodos principales - a veces utilizados por separado, a veces en combinación- mediante los cuales los candidatos políticos pueden ser admitidos o incluidos en la boleta electoral de su partido político. El primer método o sistema consiste en una simple declaración de candidatura, casi invariablemente combinada con una cuota de registro (que puede ascender a miles de dólares cuando se trata de puestos de jerarquía o importancia, con el fin de desalentar a candidatos frívolos). El segundo sistema o método consiste en una petición por parte de un grupo o de un conjunto de electores. Finalmente, el tercer método consiste en una designación a través de una convención oficial del partido en el estado de que se trate.

\section{El sistema electoral de mayoría relativa vs. la segunda vuelta}

En Estados Unidos de América, sí existe un gran número de candidatos en busca de un solo puesto de elección popular, el ganador puede serlo con un porcentaje muy pequeño

\footnotetext{
${ }^{11}$ http://grassrootsidgob.wordpress.com/list-of-states-with-open-and-closed-primaries/.

12

http://jstor.org/discover/10.2307/1025601 uid=3738664\& uid=2129\&uid=2\&uid=70\&uid=4\&sid=21103031833 007.

${ }^{13}$ http://www.fvap.gov/resourses/media/vaghandout1.pdf.

${ }^{14}$ http://Www.fvap.gov/resources/media/vaghandout1.pdf.
} 
Esta obra forma parte del acervo de la Biblioteca Jurídica Virtual del Instituto de Investigaciones Jurídicas de la UNAM www.juridicas.unam.mx

del voto total. En el norte de la unión americana, casi sin excepción, la nominación le corresponde al candidato que gana la primaria, sin importar que tan pequeño sea su porcentaje de votos. En el sur, sin embargo, la elección primaria demócrata a menudo equivalía a la elección real —siendo la elección subsecuente una mera formalidad- por lo que la mayoría de los estados decidieron requerir una segunda vuelta electoral si ningún candidato lograba obtener una victoria con la mayoría absoluta en la primera vuelta primaria. Bajo la segunda vuelta electoral compiten los dos candidatos con mayor cantidad de votos obtenidos en la primera vuelta primaria. Un total de once estados actualmente tienen normas para regular la segunda vuelta (runoff elections) en elecciones primarias y son Alabama, Arkansas, Georgia, Louisiana, Mississippi, North Carolina, Oklahoma, South Carolina, Dakota del Sur, Texas y Vermont. ${ }^{15}$

La elección primaria de segunda vuelta puede ser entendida como una institución conveniente para asegurar que la persona nominada o seleccionada goce de una amplia base de apoyo en el partido político. Sin embargo, también hay que reconocer que la elección primaria de segunda vuelta ha sido despreciada y criticada fuera del sur de Estados Unidos de América, porque es costosa, tanto para los candidatos como para el público en general. En todo caso, un candidato normalmente logra obtener una clara mayoría en la primera vuelta primaria.

\section{Las elecciones primarias no partidarias}

En los estados de Nebraska y de Minnesota, ${ }^{16}$ por ejemplo, históricamente muchos regentes, funcionarios locales urbanos y jueces, así como legisladores estatales, son elegidos bajo un marco normativo no partidista. En muchos casos, la ley contempla que un candidato es elegido automáticamente, si éste recibe la mayoría absoluta en la primera vuelta primaria. Pero si no se da una mayoría, entonces los dos candidatos que reciben la mayor cantidad de votos - aunque ambos pertenezcan al mismo partidoparticipan en la elección general.

Usualmente, las elecciones no partidarias (non partisan) no son en realidad apartidarias. Tanto republicanos como demócratas promueven a sus propios candidatos y el resultado refleja la orientación política general de la zona (al igual que en cualquier

\footnotetext{
${ }^{15}$ http://www.ncsl.org/research/elections-and-campaigns/primary-runoffs.aspx.

${ }^{16}$ http://prq.sagepub.com/content/54/1/7.abstract.
} 
Esta obra forma parte del acervo de la Biblioteca Jurídica Virtual del Instituto de Investigaciones Jurídicas de la UNAM www.juridicas.unam.mx

parte del mundo o como pasa en México en la elección, por ejemplo, de consejeros electorales del IFE o del actual INE).

\section{El sufragio}

La desaparición de requisitos como el de la propiedad, los impuestos y otras exigencias atadas al sufragio (voto censitorio), también se aplican a elecciones tanto primarias como generales. Pero, después de la etapa de la "reconstrucción", en el sur de Estados Unidos de América se dio la circunstancia de que existía un sistema de partido único bajo el cual la nominación democrática equivalía de hecho a tener la elección asegurada. Los demócratas del sur idearon un sorprendente arsenal de obstáculos para excluir a los afroamericanos de las elecciones, aunque posteriormente, a través de la aplicación de la 14a. enmienda, con sus garantías de igualdad de las leyes para todos los ciudadanos, dichos estatutos y sus normas restrictivas gradualmente han sido reemplazados o invalidados.

La Ilamada "cláusula del derecho al voto adquirido", ${ }^{17}$ que otorgaba el voto únicamente a quienes habían podido votar en un año anterior específico, o a sus descendientes, fue invalidada por la Suprema Corte en 1915, en el caso de Guinn vs. US. Por otro lado, la llamada "primaria blanca" (bajo la cual el Partido Demócrata fue declarado una organización privada que podía excluir a quienes quisiera) fue declarada anticonstitucional en 1944 , en Smith vs. Allright. ${ }^{18}$ Por otro lado, el famoso poll tax impuesto para poder ejercer el voto- tal como se aplicaba a elecciones primarias que antecedían a elecciones generales para obtener puestos federales, fue abolido mediante la 24a. enmienda, ratificada en 1964. Finalmente, la Ley de Derechos Electorales (Voting Rights Act) de 1965 estableció que tanto la "prueba de alfabetismo" como el "impuesto al voto" eran figuras restrictivas inválidas si se utilizaban de una forma discriminatoria.

\section{La elección primaria presidencial}

Con el paso del tiempo, grupos de ciudadanos progresistas decidieron que la institución de la elección primaria también podía ser utilizada para romper con el poder político de las llamadas "máquinas políticas" que nombraban a los delegados a las convenciones nacionales de nominación presidencial. De esta forma, el Estado de Florida introdujo su

\footnotetext{
${ }^{17}$ http://digital.library.okstate.edu/encyclopedia/entries/G/GU001.html.

${ }^{18}$ http://www.naacpldf.org/case/smith-v-al/wright.
} 
Esta obra forma parte del acervo de la Biblioteca Jurídica Virtual del Instituto de Investigaciones Jurídicas de la UNAM www.juridicas.unam.mx

primera elección primaria presidencial en 1901, pero Wisconsin aprobó una Ley más completa bajo el liderazgo del gobernador Robert La Follete y de la legislatura dominada por los progresistas en $1905 .{ }^{19}$ El Estado de Oregón en 1910 fue más allá de la simple elección de delegados por el pueblo, y adoptó la llamada primaria presidencial preferencial (presidential preference primary), ${ }^{20}$ bajo la cual los electores indican directamente a quien preferían para la nominación de su partido.

La corriente favorable a las primarias presidenciales recibió incluso mayor impulso después de la elección de 1912, durante la cual los seguidores del presidente Theodore Roosevelt denunciaron que la nominación republicana le había sido robada a éste, por parte de republicanos de la "vieja guardia" (dinosaurios en Estados Unidos de América, a principios de siglo) que controlaban las convenciones estatales en las cuales los delegados simpatizantes del presidente Taft fueron seleccionados. Para 1916, se habían ya aprobado leyes sobre elecciones primarias presidenciales en 26 estados.

Ante el declive de la era "progresista", el interés en las primarias presidenciales declinó. De esta forma, los candidatos tendieron a menospreciar a las primarias presidenciales durante los años veinte y treinta; la razón que explicaría lo anterior podría ser el gran conflicto republicano acaecido en 1920, en el cual tres aspirantes que llevaban la delantera compitieron vigorosamente en las primarias, para que al final del día se diera el desarrollo de una situación de empate, o impasse, que hizo que la nominación finalmente le fuera otorgada a Warren Harding, de Ohio, el hombre seleccionado por los "dinosaurios" del partido, precisamente bajo la sombra de "una encerrona", metáfora usada por los estadounidenses para referirse a una "movida tras bambalina", a puerta cerrada. A la postre, Harding fue seleccionado bajo lo que en la ciencia política estadounidense se conoce como el "clásico error de Harding", expresión que se utiliza para referir una situación en la que un candidato de apariencia física inusualmente atractiva y de porte aparentemente presidencial resulta en la práctica un político deficiente. $^{21}$

\section{Evolución a partir de la Segunda Guerra Mundial}

\footnotetext{
${ }^{19}$ http://Www.u-s-history.com/pages/h988.htm/.

${ }^{20}$ http://www.utm.edu/staff/rchestee/courses_folder/Political_Science_381/PresidentialPrimaries.htm/.

${ }^{21}$ http://www.hyperink.com/Summary-Of-Charper-3-The-Warren-Harding-Errorwhy-We-Fall-For-Tall-DarkAnd-Handsome-Men-b70a36.
} 
Esta obra forma parte del acervo de la Biblioteca Jurídica Virtual del Instituto de Investigaciones Jurídicas de la UNAM www.juridicas.unam.mx

La primaria presidencial creció en importancia después de la Segunda Guerra Mundial. En 1948, el exgobernador de Minnesota, Harold Stassen, obtuvo varias victorias primarias cruciales iniciales, y muy bien pudo haber derrotado a sus rivales más prestigiados —el gobernador de Nueva York, Thomas Dewey y al senador Robert Taft, de Ohio, si no hubiera cometido errores muy serios en la contienda clave del Estado de Oregón-. ${ }^{22}$ En 1952, el senador Estes Kefauver, de Tennessee, sorprendió al presidente Harry Truman en New Hampshire. Ese mismo año, victorias en elecciones primarias logradas por el general Dwight Eisenhower, le permitieron remontar la ventaja que tenía Taft en la contienda por la nominación republicana.

En 1960, el senador John F. Kennedy, de Massachusetts, barrió en las primarias, con lo que demostró sus habilidades para obtener votos y sobreponerse a la oposición, basado en su juventud y catolicismo. Por otro lado, el senador George McGovern, de Dakota del Sur, y el exgobernador Jimmy Carter, de Georgia, obtuvieron las nominaciones demócratas en 1972 y en 1976, respectivamente, después de haber derrotado a sus oponentes en elecciones primarias estatales claves.

Ahora bien, cabe establecer que las elecciones primarias presidenciales en Estados Unidos de América no son siempre la única vía hacia la Casa Blanca. Kefauver logró salir triunfante de la mayoría de las elecciones primarias en 1952, pero no fue nominado. El vicepresidente Hubert $\mathrm{H}$. Humphrey se mantuvo alejado de las primarias en 1968, pero tuvo la nominación presidencial de su partido a pesar de todo. De esta forma, un candidato altamente aceptable para los líderes regulares de la organización de su partido puede ganar sin las primarias. Pero, por otro lado, un candidato que carece de apoyo organizativo no tiene otra alternativa que entrar a las primarias y realizar un buen papel en éstas, si es que desea ser nominado. Aun así, en este último caso, éste puede ser derrotado o ver disminuidas sus posibilidades, si la nomenclatura del partido se opone a su candidatura —-destino que le esperaba a Kefauver-.

\section{El derecho estadounidense vigente}

En los últimos lustros, diversas reformas electorales del Partido Demócrata han llevado a un aumento en el número de elecciones presidenciales primarias que ya supera las 30 , siendo en 2012 exactamente 36 las entidades federativas de Estados Unidos de América

\footnotetext{
${ }^{22}$ http://www.nytimes.com/2001/03/05/us/harold-e-stassen-who-sought-gop-nomination-for-president-9times-dies-at-93.html?pagewanted=al/\&src $=p m$.
} 
Esta obra forma parte del acervo de la Biblioteca Jurídica Virtual del Instituto de Investigaciones Jurídicas de la UNAM www.juridicas.unam.mx

que celebran elecciones primarias presidenciales y 14 que usan la figura de los caucuses: Alaska, Colorado, Hawaii, Idaho, Iowa, Kansas, Maine, Minnesota, Missouri, Nevada, North Dakota, Utah, Washington y Wyoming. ${ }^{23}$

Existen básicamente dos tipos de elecciones presidenciales primarias. En el voto de preferencia (preference pol/), los electores anotan su selección de los nominados en la boleta electoral. Alternativamente, o concurrentemente, los electores pueden seleccionar a todos o a parte de su delegación estatal a la convención de nominación en la primaria. Las reglas del Partido Demócrata han exigido que los delegados sean elegidos con un criterio proporcional, eliminando, de esta manera, la característica de que "el que gana, se lleva todo" (winner takes all) de los primeros años, práctica que, sin embargo, existe y es legal todavía en las primarias republicanas.

Ahora bien, los anteriores cambios a las reglas del Partido Demócrata, junto con las reformas a las leyes federales que en su momento se dieron en materia de financiamiento de campañas, que limitaban los montos que un donador puede contribuir y la cantidad que un candidato puede gastar en las campañas "preconvención", han ayudado fundamentalmente a los candidatos más débiles, o menos poderosos, que de esta forma pueden escoger estados en los que pueden gastar la menor cantidad para tener el mejor resultado y de esta manera ganar impulso para futuras primarias.

Ahora bien, debido a que tradicionalmente se celebra en primer término (enero 10 de 2010, en la última elección) la primaria de New Hampshire, era usualmente la que durante mucho tiempo atraía la mayor atención mediática, aunque ahora la primera es la de Iowa, celebrada el 3 de enero de 2012. Por otro lado, Florida, Wisconsin, Oregón, Nebraska, Maryland, North Carolina, Texas, Tennessee, Masachusetts, Indiana y Pennsylvania también atraen mucha atención como "pruebas" de popularidad en regiones con distintas características geográficas y electorales. La temporada continúa hasta principios de junio, cuando se celebran las primarias importantes de California y New Jersey.

\section{Los argumentos en pro y en contra}

de las primarias presidenciales

Existen críticos que argumentan que las elecciones primarias presidenciales a menudo

${ }^{23}$ http://uspolitics.about.com/od/presiency/a/Primary-Dates-In-The-2012-Presidential-Contest.htm. 
Esta obra forma parte del acervo de la Biblioteca Jurídica Virtual del Instituto de Investigaciones Jurídicas de la UNAM

polarizan ideológicamente a los miembros de los partidos políticos; que no existe un consenso realmente nacional que pueda emanar de éstas; que agotan las energías de los candidatos en giras de "saludos de manos" irrelevantes; que distraen la atención de los asuntos y las cuestiones primordiales y que cuestan cantidades injustificables de dinero. Por otro lado, quienes promueven y apoyan las elecciones primarias contestan que éstas posibilitan la existencia de cuando menos una oportunidad real para que el elector común exprese su opinión sobre los candidatos potenciales, sin que los grandes "jefes" (bosses) del partido hablen por él, y son además un terreno fértil de experimentación y prueba vital que demostrará qué candidatos poseen la energía y la calma necesarias, bajo condiciones prácticas de presión, que se esperan de quien podría llegar a ser presidente. Posiblemente, la contribución más importante de las primarias es el hecho de que ponen fin - rápida y contundentemente- a candidaturas carentes de base, frívolas o insostenibles.

Cabe señalar que junto con las encuestas de opinión nacionales en los años de elección presidencial, las primarias han dificultado la posibilidad de que en las convenciones nacionales se haga caso omiso de los deseos y de la voluntad de los miembros de base de un partido. Por otro lado, la televisión otorga una publicidad fundamental para las campañas de los candidatos en los estados que contemplan elecciones primarias. Con frecuencia, un líder natural poderoso y fuerte surge del proceso de nominación antes de celebrarse la Convención, y los delegados se sienten obligados a nominarlo. Las situaciones de empate técnico o impasse en las convenciones —hoy día - son raras, y por otro lado, casi nunca respaldan a un "caballo negro".

\section{4. ¿Qué es preferible: elecciones primarias presidenciales}

\section{regionales o nacionales?}

Desde que, por primera ocasión, el presidente Woodrow Wilson propuso la idea en 1913, se ha presentado una discusión y debate esporádicos sobre la posibilidad de una elección primaria presidencial nacional. Bajo este supuesto, las convenciones nacionales de los partidos, de celebrarse, ya no nominarían a candidatos.

Otros expertos más favorecen una serie de primarias regionales celebradas cada año, que reducirían notablemente los viajes a lo largo y ancho de Estados Unidos de América de los candidatos. Pero quienes se oponen a esta idea, dicen que existe el riesgo de que aquéllos candidatos que sean viables en tan sólo una sección del país podrían emerger 
Esta obra forma parte del acervo de la Biblioteca Jurídica Virtual del Instituto de Investigaciones Jurídicas de la UNAM

con los ganadores.

Quienes proponen celebrar una elección primaria nacional dicen que ésta permitiría al pueblo decidir directamente respecto a un candidato o nominado presidencial de su partido, cortando de tajo la posibilidad de que manipuladores políticos usen su influencia sobre el propio proceso de nominaciones para la obtención del puesto de elección popular de más jerarquía en el país.

Por otro lado, quienes se oponen, sostienen que la convención nacional juega un papel positivo al obligar a los delegados a decidir respecto a una plataforma y a una fórmula para presidente y vicepresidente que sea aceptable a la mayoría de las corrientes y alas del partido en conflicto, de esta forma, llevan a efecto internamente los compromisos necesarios para el funcionamiento de un sistema bipartidista como el estadounidense.

\section{ELECCIONES INTERNAS DEMOCRÁTICAS Y SUS EFECTOS SOBRE LOS PARTIDOS POLÍTICOS ESTADOUNIDENSES}

\section{Las primarias reguladas por leyes estatales}

\section{como instituciones de derecho público}

Para que las elecciones generales se puedan celebrar bajo condiciones adecuadas de transparencia y equidad, y con el mínimo de quejas, enfrentamiento e incluso violencia, debe existir un control sobre los mecanismos electorales de selección de los candidatos y dirigentes políticos. A diferencia de aquellos países de Europa occidental que permiten a los propios partidos políticos regular los procesos electorales internos y primarios, cabe destacar de entrada que los partidos políticos estadounidenses no tienen abierta esta posibilidad (este señalamiento es de suma importancia, puesto que si en México tanto el propio PRI como algunos partidos actualmente de oposición planean implementar elecciones primarias "a la estadounidense" para seleccionar a su candidato presidencial para 2018, habría que ponderar qué implicaciones tiene esta acción en un país en que los procesos electorales internos de los partidos no están regulados integralmente en detalle, ni en leyes electorales estatales ni en regulaciones federales).

Así, en Estados Unidos de América, los estados —en su ámbito competencial— son los encargados de regular a los partidos, que son considerados como entidades con una naturaleza pública; si bien se perciben algunos aspectos privados, los intereses y recursos que los partidos políticos representan tienen repercusiones públicas muy por 
Esta obra forma parte del acervo de la Biblioteca Jurídica Virtual del Instituto de Investigaciones Jurídicas de la UNAM

encima de las estrictamente privadas. De esta forma, las leyes son reformadas frecuentemente debido que los legisladores buscan desarrollar o establecer el mejor sistema posible. Así pues, bajo este espíritu estadounidense, los procesos electorales son un asunto de importancia y de repercusión pública, y por lo tanto, deben ser manejados en el ámbito de lo público, y no en cuartos cerrados, esto es a puerta cerrada por parte de élites partidistas.

El anterior contexto impulsó las reformas progresistas ya citadas en este estudio a principios del siglo XX, que abrieron a los partidos políticos a la sociedad y eliminaron la política de los llamados "cuartos cerrados". Estos cambios incluyen, entre otros temas, reformas que han establecido iniciativas populares (medidas legislativas iniciadas por los electores, no por los legisladores), referendos (votaciones a nivel estatal, usualmente llevadas a cabo por las legislaturas estatales) y la revocación de mandato (peticiones para remover a un funcionario de su puesto), así como otros cambios que fueron evidentes en los años sesenta y setenta para abrir los partidos políticos - particularmente al Partido Demócrata- a las mujeres y a las minorías. Por otro lado, ante la maniobra de los partidos políticos que han desplazado la selección de delegados a las convenciones, las elecciones primarias se han incrementado. Estos procesos electorales son regulados en lo fundamental por los estados, por lo que los partidos se adaptan a sus regulaciones.

Cabe resaltar que el papel de los partidos políticos en la nominación de candidatos políticos, se encuentra limitado por requisitos legalmente impuestos a través de las leyes electorales estatales, en especial por la llamada primaria directa como el método aprobado y "certificado" para escoger a los candidatos de los partidos. La elección primaria y el apartidismo son factores que abren los procesos de selección de candidatos a un involucramiento del público en general, lo que permite que incluso miembros "eventuales" u ocasionales y casuales de los partidos, escojan a los nominados que la entidad partidaria debe aceptar como propios.

Estos procedimientos se definen como "democráticos" en el sentido de que permiten a una mayor cantidad de ciudadanos tener la oportunidad de involucrarse en la política. Ahora bien, el que mediante estos métodos se abran los procesos de nominación a una verdadera avalancha de candidatos y de electores igualmente carentes de experiencia, y que el debate sobre si los partidos en realidad deberían ser soberanos o deben compartir sus facultades con el electorado es -en la actualidad- sin duda, cuestionable. 
Esta obra forma parte del acervo de la Biblioteca Jurídica Virtual del Instituto de Investigaciones Jurídicas de la UNAM

En el anterior sentido, no cabe duda que los líderes juegan un papel importante, aunque menos significativo, en aquéllas arenas y temas en las que el partido es competitivo.

En realidad, la pregunta fundamental sería ¿qué tan "democráticos" deben ser los partidos políticos en una democracia?, ¿deberían ser los líderes de los partidos - la nomenclatura- quienes seleccionan a los candidatos, o debe ser la "masa" del electorado quien tome dicha decisión? En este sentido, en Estados Unidos de América parece haber la tendencia de permitirle al electorado seleccionar a los candidatos, promoviéndose, por lo tanto, valores democráticos por encima de los de la eficiencia partidaria.

Por otro lado, ¿acaso son distintos los candidatos que son resultado de un proceso en el cual son los líderes y no el electorado los que realizan la selección? Es difícil contestar a dicha pregunta debido a que el proceso es "interactivo". Si bien las elecciones primarias permiten que opciones no partidarias tengan juego, no cabe duda que los líderes y dirigentes partidarios sí desempeñan un papel en áreas en las que el partido es competitivo -aunque no el papel decisivo que en alguna época llegaron a desempeñar-.

Una crítica frecuente, vinculada a las derrotas sufridas por los candidatos demócratas en las elecciones presidenciales en los años ochenta, surgía de la sensación persistente de que ampliar y abrir los procesos de selección no resultaba necesariamente en candidatos victoriosos en la elección general.

¿Bajo qué condiciones es que tiene mayor impacto la elección primaria directa sobre el control de la organización partidaria? La respuesta está vinculada al fenómeno del llamado "unipartidismo": un partido en particular domina de tal manera la elección general que cualquier candidato seleccionado por dicho partido virtualmente será siempre el ganador. De esta forma, la principal campaña de promoción progresista de la llamada primaria abierta se dio durante el periodo del unipartidismo en la historia estadounidense (aunque a nivel nacional, los partidos sí eran competitivos).

De esta manera, la estructura partidista aún conserva el control de las nominaciones locales en aquellas demarcaciones en donde el partido es relativamente fuerte, disciplinado, integrado, leal y por lo general ejerce el control de los dineros y los recursos necesarios para dominar la elección primaria a través de apoyos y asistencia para 
Esta obra forma parte del acervo de la Biblioteca Jurídica Virtual del Instituto de Investigaciones Jurídicas de la UNAM www.juridicas.unam.mx

realizar campañas electorales. Pero los grupos de interés y las "personalidades" que compiten con las estructuras partidarias (caso de Fox y el PAN en 2000 y posteriormente de AMLO en 2006 y 2012) disponen de recursos similares y compiten directamente por los apoyos en las elecciones primarias.

\section{Las elecciones primarias no siempre garantizan una efectiva}

participación de las bases o masas en los procesos

de selección de candidatos

Al final del día, aunque las elecciones primarias facilitan una oportunidad para la participación popular en los procesos internos de selección de candidatos, las masas del electorado muy rara vez están lo suficientemente interesadas para aprovechar el poder político potencial que les presentan las elecciones primarias para darse a sí mismo mejores opciones en las elecciones generales. Cabe resaltar que la votación en las elecciones primarias es notoriamente baja en Estados Unidos de América; incluso, en las elecciones presidenciales, las primarias promedian únicamente el $25 \%$ de participación de los electores empadronados, por lo que su futura implementación en México deberá incluir mecanismos para generar interés de la ciudadanía en dichas elecciones internas para evitar su fracaso prematuro.

\section{Algunos prolegómenos de las elecciones}

primarias presidenciales

El proceso de las elecciones presidenciales en Estados Unidos de América se inicia cada vez más temprano, debido a que los candidatos potenciales se comienzan a posicionar favorablemente dentro del partido y ante los medios masivos hasta con dos años de anticipación a la fecha de la elección (nuevamente vienen a la mente los casos de Vicente Fox y el PAN en 2000, en el cual la hiperactividad de Vicente Fox prácticamente "secuestró" la candidatura ante la nomenclatura expectante del PAN tradicional, y de Andrés Manuel López Obrador y su recorrido por el territorio nacional con años de anticipación a las elecciones presidenciales de 2006 y 2012). De esta forma, las ambiciones por obtener la presidencia pueden tener su raíz en el pasado, pero la planeación estratégica, de hecho, comienza para los candidatos serios a menudo inmediatamente después de la última elección —en el caso, podríamos decir que en realidad en este contexto no sale quien no se mueve (esta etapa normalmente se encuentra fuera de la observación de los medios masivos, aunque debe resaltarse el 
Esta obra forma parte del acervo de la Biblioteca Jurídica Virtual del Instituto de Investigaciones Jurídicas de la UNAM www.juridicas.unam.mx

fenómeno de Andrés Manuel López Obrador y Morena, pues rumbo a la elección de 2018 está aprovechando hábilmente, y en solitario por el momento, los defectos de diseño institucional de las normas electorales vigentes que le permiten una exposición mediática adelantada)—y más abiertamente, unos dos años después de la misma.

De esta forma, volviendo al ámbito estadounidense, los candidatos comienzan a hacer públicas sus intenciones de manera formal seis meses antes de las juntas o reuniones del liderazgo partidario de Iowa; los candidatos para la nominación republicana de 1996, por ejemplo, comenzaron a difundir su aspiración a la candidatura presidencial casi con un año de antelación a la celebración de la primera vuelta primaria o la primera junta o reunión de liderazgo.

De hecho, durante la etapa de campaña, los diversos candidatos parecen participar en un juego de expectativas. De esta manera, si declaran que esperan acercarse levemente a quien en ese momento va adelante en las encuestas e incluso alcanzarlo, entonces, repentinamente los medios masivos presentan a quién va delante como candidato "en problemas", y al que le sigue se le ve como una amenaza mayor de lo que los números y estadísticas indicarían. Por ejemplo, este es el caso de 1984, de Gary Hart, que cerró la distancia que le separaba de Walter Mondale en la primaria de New Hampshire; y en 1988, de Pat Robertson y Bob Dole que se desempeñaron muy bien ante el entonces vicepresidente George Bush en los caucuses de Iowa. Sin embargo, como posteriormente se pudo apreciar, estos pequeños éxitos estaban totalmente fuera de proporción con la realidad profunda de cada candidato.

En el anterior sentido, es que existen varias preguntas y cuestiones en relación con el tema de cuál es el mejor mecanismo para seleccionar al presidente, y cada elección acarrea modificaciones al sistema y una andanada de propuestas de reforma, caso similar a lo que está sucediendo en México en las últimas tres elecciones presidenciales.

En primer término, ¿por qué es que los caucuses de Iowa y las primarias de New Hampshire tienen un gran impacto tan decisivo en la selección de candidatos? Si ninguno de los dos estados es particularmente típico. En Iowa, por ejemplo, los candidatos enfatizan los asuntos agrarios y rurales, que no conciernen a los electores en los estados de corte netamente urbano. Claro está que cronológicamente cualquiera que sea el estado en el que se celebren primero las elecciones primarias, éste siempre tendrá por ese solo hecho un peso específico crucial en la campaña que no es aplicable al resto de 
Esta obra forma parte del acervo de la Biblioteca Jurídica Virtual del Instituto de Investigaciones Jurídicas de la UNAM

DOI: http://dx_doï.org/10.22201/iij.24487910e_2016.9.10096

la nación. Este es un asunto que habrá que cuidar mucho en un futuro, en México, de implementarse el sistema de elecciones primarias para las elecciones presidenciales, y que podría servir para realizar un ejercicio de "ingeniería electoral". De esta forma, si en el Estado de Iowa, por ejemplo, lo que importa son los asuntos agrarios, entonces serían los asuntos forestales en Oregón, o la pesca en el estado de Maine, los temas que predominarían. El quid del asunto es enfatizar los temas regionales vinculados a la geografía, el comercio e incluso la historia inherentes al estado de que se trate, con el fin de lograr un efecto de amplificación del impacto obtenido en dicha entidad en el ámbito nacional, que siempre concentrará su atención en los resultados y estadísticas de la primera elección primaria como una suerte de "laboratorio" de lo que pasará en el resto del país.

Por otro lado, hay razones financieras y de equidad en Estados Unidos de América por las cuales los estados más grandes como California, o como Nueva York no celebran las primeras elecciones primarias, puesto que la necesidad de realizar una campaña intensa para tener los delegados de esos estados al inicio del proceso electoral, eliminaría a los candidatos menos conocidos y con menor poder económico que no podrían implementar los esfuerzos logísticos y mediáticos necesarios para tener éxito en demarcaciones demasiado grandes geográfica y poblacionalmente. Por otro lado, los demócratas en Estados Unidos de América están comenzando a percatarse que el poner atención especial a las necesidades del Norte (New Hampshire, por ejemplo) los deja ante la imposibilidad de obtener una victoria en el más conservador sur. En el caso de México, los problemas de marginación en el sureste nacional, y las quejas del Norte respecto al centro del país también originarán estrategias propias del país, de implementarse a futuro un sistema de elecciones primarias presidenciales.

\section{El dilema de las primarias regionales}

\section{$y$ de las primarias nacionales}

Ante el dilema de las elecciones primarias regionales y nacionales, cabe establecer que ambas opciones ofrecen ventajas y desventajas potenciales, y ambas deberán ser consideradas a futuro por los partidos políticos en México, puesto que implican resultados y dinámicas distintas; así, una serie de primarias regionales puede diluir o matizar ciertas tendencias electorales, que se presentarían con gran claridad de celebrarse primarias estatales en todas las entidades, o bien una sola primaria nacional. 
Esta obra forma parte del acervo de la Biblioteca Jurídica Virtual del Instituto de Investigaciones Jurídicas de la UNAM

DOI: http://dx_doï.org/10.22201/iij.24487910e_2016.9.10096

Una elección primaria nacional evitaría que un solo estado, como tal, tuviera una influencia excesiva al inicio de las campañas, pero, por otro lado, probablemente implicaría que los candidatos realizarían sus campañas tan sólo en los estados más grandes. Los estados más pequeños serían "descuidados", debido a que los candidatos deben procurar utilizar los recursos disponibles inteligente y estratégicamente; esto es, deben optimizarlos para obtener la mayor cantidad de delegados posible. Por otro lado, también sería necesario que los candidatos estuvieran financiados robustamente desde el inicio, debido a que la inercia de campaña - la dinámica de campaña- no dependería de un crecimiento paulatino - que favorece a los más débiles económicamente- a través de una serie de primarias estatales.

Por otro lado, hay que argumentar que la anterior práctica acortaría las campañas, y por ende ayudaría a que los candidatos enfocaran su atención sobre las grandes cuestiones nacionales, y no sobre la propia contienda. Por otro lado, están las encuestas de opinión y electorales que reforzarían el aspecto competitivo, por lo que las "personalidades" fuertes se verían aún más enfatizadas. Una posibilidad positiva para los partidos políticos podría ser el que los candidatos podrían ser seleccionados a través de una convención, como resultado de que ningún candidato en lo particular recibiera una mayoría clara. De esta manera, el papel de los partidos políticos podría fortalecerse bajo un sistema de primarias nacionales. Por otro lado, el papel de los partidos políticos podría disminuir, incluso aún más, si la primaria nacional proclamara a un ganador con excesiva rapidez y facilidad.

Por otra parte, una serie de primarias regionales (vis a vis primarias estatales) permitiría alguna de las ventajas de las contiendas grupales y un calendario menos riguroso de elecciones, con la subsecuente posibilidad para los candidatos en desventaja, por falta de recursos para poder construir poco a poco la dinámica de la campaña. De hecho, las primarias de 1988 y de 1992 en Estados Unidos de América son clara evidencia de la realización de los primeros intentos en esta dirección.

Por último, existe una cuestión de la mayor relevancia: la tendencia a que la mayoría de los estados de la unión americana se incline hacia las primarias (y se alejen simultáneamente de las juntas o reuniones cupulares o de liderazgo (caucuses), como las que se celebran en Minnesota), y el hecho de que los candidatos a la presidencia de Estados Unidos de América disponen actualmente de estructuras electorales personales (caso que se dio con Fox en México en las elecciones de 2000). En este sentido, ¿cuál será 
Esta obra forma parte del acervo de la Biblioteca Jurídica Virtual del Instituto de Investigaciones Jurídicas de la UNAM www.juridicas.unam.mx

-a futuro- el papel de la figura del partido político tradicional ante los procesos de nominación presidencial tanto en Estados Unidos de América, como a futuro en México?

\section{ALGUNAS CONSIDERACIONES FINALES}

1. Con base en las reflexiones realizadas a lo largo del presente estudio sobre la evolución de la figura de las elecciones primarias bajo el modelo estadounidense como elecciones preliminares que anteceden a las elecciones generales para ocupar cargos a nivel municipal, estatal y federal, podemos concluir que dicho método de selección de candidatos y líderes políticos han democratizado indudablemente el acceso al poder político en Estados Unidos de América, en contraste con procedimientos de selección más restrictivos y opacos como las convenciones, las encuestas o los Ilamados "dedazos".

2. Adicionalmente, en ámbitos en que la delincuencia organizada constituye un acto de poder y de influencia que rivaliza con el propio estado -legal y legítimamente constituido-, las elecciones primarias disminuyen la posibilidad de narcocandidatos que acceden exitosamente al poder a través de elecciones constitucionales, "lavando" así su capital político.

3. Por otro lado, al sortearse los escándalos inevitables con el descubrimiento tardío de narcopolíticos, ya en ejercicio del poder, surgidos de procesos electorales "opacos", se protege al propio sistema de partidos políticos y su reputación institucional como única vía aceptable de acceso al poder político constitucionalmente legal y legítimo.

4. Asimismo, si la "responsabilidad" de la elección de un candidato vinculado a la delincuencia organizada descansa en el electorado que no se percató a tiempo de las deficiencia o legitimidad de origen de un candidato, el daño a la idea misma de la democracia y las elecciones es menor que el que se observa cuando los narcopolíticos o gobernantes vinculados a la delincuencia organizada surge de métodos de selección restrictivos y cerrados a la participación transparente y popular.

5. Por último, debe recalcarse que los partidos políticos son instituciones fundamentalmente para la estabilidad política y social de cualquier país, y del propio sistema político y de partidos. Por ello, casos como el del narcocandidato José Luis Abarca dañan no sólo a un partido a nivel local sino a la dirigencia nacional de dicho partido, y por ende al equilibrio del sistema de partidos políticos en México. 